\title{
Ground Penetrating Radar (GPR) Investigations for Architectural Heritage Preservation: The Case of Habib Sakakini Palace, Cairo, Egypt
}

\author{
Sayed Hemeda \\ Conservation Department, Faculty of Archaeology, Cairo University, Giza, Egypt \\ Email: hemeda@civil.auth.gr
}

Received April 14, 2012; revised May 18, 2012; accepted May 28, 2012

\begin{abstract}
A comprehensive Ground Penetration Radar (GPR) investigations and hazard assessment for the rehabilitation and strengthening of Habib Sakakini's Palace in Cairo is presented herein, which is considered one of the most significant architectural heritage sites in Egypt. The palace located on an ancient water pond at the eastern side of Egyptian gulf besiding Sultan Bebris Al-Bondoqdary mosque is a place also called "Prince Qraja al-Turkumany pond”. That pond had been filled down by Habib Sakakini at 1892 to construct his famous palace in 1897. The integrated geophysical survey of the palace allowed the identification of several targets of potential archaeological and geotechnical engineering interest buried in fill and silty clay in the depth range between $100-700 \mathrm{~cm}$. the methodological development focused on Multi-Fold (MF) Ground Penetrating Radar (GPR) imaging and subsurface characterization based on integrated velocity and attenuation analysis. Eight hundred sqm of Ground penetration Radar (GPR) profiling have been conducted to monitor the subsurface conditions. 600 meters are made in the surrounding area of the Palace and 200 sqm at the basement. The aim is to monitor the soil conditions beneath and around the Palace and to identify potential geological discontinuities, or the presence of faults and cavities. A suitable single and dual antenna are used (500 - $100 \mathrm{MHZ}$ ) is used to penetrate the desired depth of 7 meters (ASTM D6432). The GPR is used also detect the water table. At the building basement the GPR is used to identify the foundation thickness and soil-basement interface. As well as the inspection of cracks in some supporting columns, piers and masonry walls. The GPR also was used to investigate the floors and ceilings conditions and structural mapping. The results were validated by the geotechnical and structural surveys. All these results together with the seismic hazard analysis will be used for the complete analysis of the palace in the framework of the rehabilitation and strengthening works foreseen in a second stage.
\end{abstract}

Keywords: Ground Penetration Radar (GPR); Architectural Heritage Preservation; Site Investigations; Geophysics; Restoration of Monuments

\section{Introduction}

The modern architectural heritage of Egypt is rich, and extensively variable. It covers all kind of monumental structures like palaces, public buildings, residential and industrial buildings, bridges, springs, gardens and any other modern structure which falls within the definition of a monument and belongs to the Egyptian cultural heritage.

The majority of these buildings are private properties, which are either part of a traditional settlement, or isolated buildings incorporated into the wider area (such as towers and konaks) or into a completely changed and entirely modern area.

Preservation of the architectural heritage is considered a fundamental issue in the life of modern societies. In addition to their historical interest, cultural heritage build- ings are valuable because they contribute significantly to the economy by providing key attractions in a context where tourism and leisure are major industries in the 3rd millennium. The need of preserving historical constructions is thus not only a cultural requirement, but also an economical and developmental demand.

The study of historical buildings and other structures must be undertaken from an approach based on the use of modern technologies and science. The final aim must be to select and adequately manage the possible technical means needed to attain the required understanding of the morphology and the structural behavior of the construction and to characterize its repair needs. Modern requirements for an intervention include reversibility, unobtrusiveness, minimum repair, and respect of the original construction, as well as the obvious functional and 
structural requirements. Restoration operations complying with these principles require a scientific, multidisciplinary approach that comprehends historical understanding, modern non-destructive inspection techniques, and advanced experimental and computer methods of analysis.

The archaeological subsurface represents a potentially difficult problem for imaging targets with ground penetrating radar (GPR) systems. Structures within the ground may be very steeply dipping or exhibit large local variations in strike causing serious migration problems. Furthermore, the target may be located in a medium that is electrically conductive (e.g., clay-rich or waterlogged soil) such that the GPR wavelet is rapidly attenuated and the signal-to-noise ratio (SNR) in the resulting dataset is diminished. Recently, techniques used routinely by the seismic industry for imaging complex, low-SNR targets have been imported to the field of GPR acquisition. Specifically, use of three-dimensional (3-D) migration has been shown to significantly improve the resolution of the GPR target in regions of structural complexity, whilst the multi-offset (MO) method has been employed to boost SNR over otherwise poorly defined targets [1]. When these methods are combined, the potential improvement to the image of an archaeological target may be considerable. An integrated 3-D, MO GPR acquisition was performed over an archaeological target, over a RomnoBritish villa at Ground well Ridge in Spring 2006.

Ground penetrating radar (GPR) is extensively used for a variety of applications. Among all the high-resolution geophysical methods, GPR has proven to be the most suitable for detection of karstic cavities and sinkholes, in a wide range of soil and rock conditions [2]. However, one of the main limitations of GPR is the exact determination of the mean velocity of the electromagnetic waves, which is a key datum to estimate the depth of penetration into the ground.

\section{Ground Penetrating Radar Survey}

The GPR functions by sending high frequency electromagnetic waves into the from a transmitter antenna. Some of these waves are reflected back to the surface as they encounter changes in the dielectric permittivity of the matrix through which they are travelling, and are detected by a receiver antenna. The amplitude and twoway travel time of these reflections are recorded on a portable computer. This information is then used to construct a two-dimensional plot of horizontal distance versus travel time. Data collected in the field are stored on a portable computer for later analysis. A more complete and technical discussion of the method can be found elsewhere $[3,4]$.

The effectiveness of GPR is controlled by the local soil conditions. GPR is most effective in locating buried objects in a homogenous soil matrix with a high electrical resistance. GPR is least effective in a heterogeneous environment with high electrical conductivity. A heterogeneous environment contributes to signal scattering and can result in insufficient depth of penetration and a "noisy" reflection (poor signal to noise ratio). A conductive environment can seriously inhibit depth of penetration due to conductive losses. Conductive loss is the result of the electromagnetic wave creating a conductive current in the soil medium, resulting in signal attenuation.

Although GPR survey can be performed in a number of ways, the method employed in this survey involves dragging the transmitter and receiver antennas together over the ground, called fixed offset reflection mode. The transmitter emits pulses at regular intervals along a transect which are picked up by the receiver. A laptop computer controls data collection and displays the data as a two dimensional profile.

The GPR is able to detect subsurface features whose electrical properties contrast with those of the surrounding soil. The GPR can detect human burials in several ways. It may detect the disturbed soil of the grave shaft, or a break in the natural stratigraphy or soil profile [5]. It may also detect the coffin, bones, clothes and other articles in the burial. Reflections may be caused by air voids within the skull [6] or the coffin. It has also been suggested that the decomposition of bones may leach calcium salts into the surrounding soil for many years, which may change the electrical properties of the soil, making it visible to the radar.

The integrated geophysical survey of the palace allowed the identification of several targets of potential archaeological and geotechnical engineering interest buried in fill and silty clay in the depth range between 100 $700 \mathrm{~cm}$. the methodological development focused on Multi-Fold (MF) Ground Penetrating Radar (GPR) imaging and subsurface characterization based on integrated velocity and attenuation analysis.

Information about geometry and location of targets is integrated by attenuation and velocity of radar waves, which provide direct evidence of varying electromagnetic properties (conductivity, dielectric constant) and allow a detailed characterization of surveyed volume based on physical characteristic of soil strata, buried foundations and materials. Applications of combined techniques can be successful in preliminary exploration of pre-historical sites, see [7] and of archaeological sites of later periods. The contrast in physical properties of the materials and the sensitivity of the different methods play a key role in applications of geophysical methods to archaeological and engineering surveys and determine the probability of success. The GPR is extensively applied to study archaeological sites, see [8-12]. 


\section{Ground Penetration Radar (GPR) Data Acquisition and Analysis}

Mala X3M Radar System coupled to shielded antenna of $500 \mathrm{MHz}$ and $250 \mathrm{MHz}$ central frequencies was used to accomplish the survey. Distances along the surveyed lines were accurately recorded using a measuring tape. The profile spacing was assumed according to the available space in the Palace, and the trace interval of 0.02 meters for the $500 \mathrm{MHz}$ antenna profiles. About 100 profiles were conducted at the site (Figures 1 and 2). The radar antenna was moved along the lines, and the $2 \mathrm{D}$ profiles of a large number of periodic reflections were generated, thus producing a profile of the subsurface structure, with a fixed gain. Special care was taken to avoid artificial high-frequency noise that could deteriorate the radar signal. Time windows of $60 \mathrm{~ns}$ and $170 \mathrm{~ns}$ were used during data acquisition in order to receive information from sources located as deep as possible. The layout of the conducted profiles in basement and first floors are shown in (Figures 1(a) and (b)).

\section{GPR Data Processing}

The analysis of GPR data is carried out by processing the data using different gains and filtering techniques. Gain is a value, by which raw data are multiplied, to enhance low amplitude reflections. Signal amplitude commonly decreases exponentially at increasing travel times (greater depths below surface). This is compensated by designing a time gain that increases the signal strength at greater travel times. Filtering is the use of mathematical processing algorithms to "clean" noises from the data and/or enhance certain characteristics of the data. This process was specifically customized for each profile. Data processing routine includes background noise removal, time-zero corrections and band-pass filtering applied to the acquired data. Given that, most of the energy is lim-

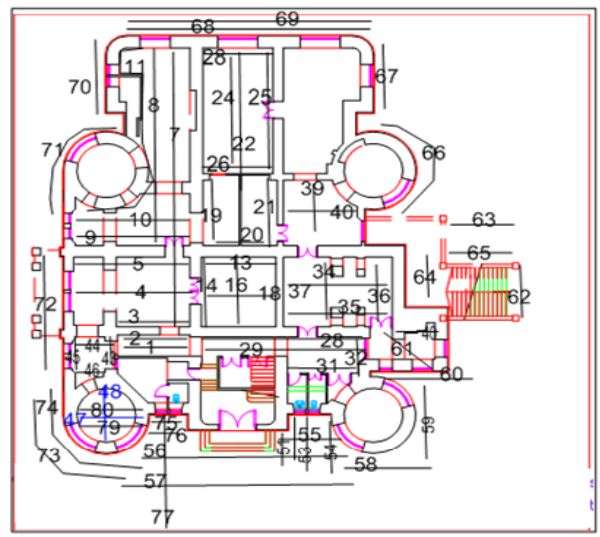

(a) ited to a finite bandwidth, an appropriate use of band limiting filtering may improve signal-to-noise without significantly altering the data [13]. Taking into account the information obtained from the amplitude spectra of the raw data, a common band pass filter of $250 \mathrm{MHz}$ to $750 \mathrm{MHz}$ was applied to the whole set of $500 \mathrm{MHz}$ profiles, and $150 \mathrm{MHz}$ - $300 \mathrm{MHz}$ band-pass filter applied to the whole set of $250 \mathrm{MHz}$ profiles to improve the signal quality.

Since the radar velocities and the dielectric properties of the studied materials were unknown, two different techniques were used to estimate the mean velocities of the electromagnetic waves: 1) determination of the velocity that produces the best fit between the measured GPR reflections and the dips and depths of the geological structures identified with seismic surveys (e.g. a lithological boundary resulting in contrasting physical properties); 2) determination of the mean velocity of the material directly related to the geometry of the hyperbolic reflections originated by point sources. The second method used in the present study where a test was carried out on a known depth objects at the site. All the data were processed, modeled and interpreted using the software Reflex_W.4 [14]. In all the profiles, the position of antenna is represented in the horizontal axis, whereas depth is depicted in the vertical one. An example of data processing results is shown in (Figure 3).

\section{Interpretation of Geophysical Survey Results}

The results of geophysical surveys of archaeological sites are generally presented graphically. This is done because anomalies of cultural origin are generally recognized by their pattern, rather than by their numeric values alone. When rendered graphically, we can better recognize cultural and natural patterns and visualize the physical phenomena causing the detected anomalies.

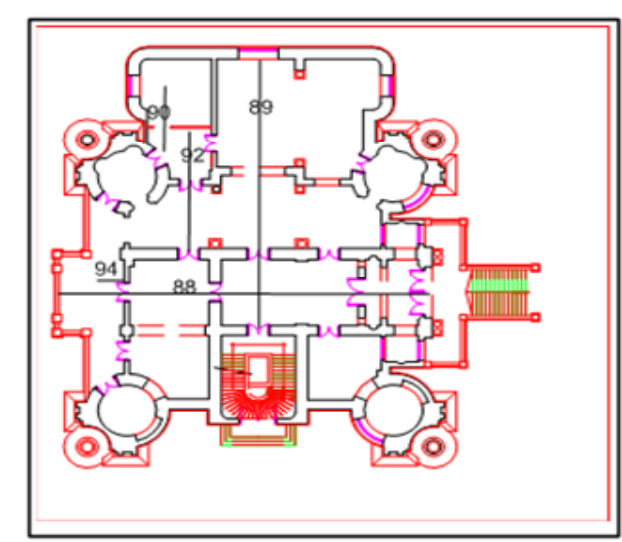

(b)

Figure 1. (a) GPR layout at basement and area around the palace; (b) GPR layout at first floor of the palace. 

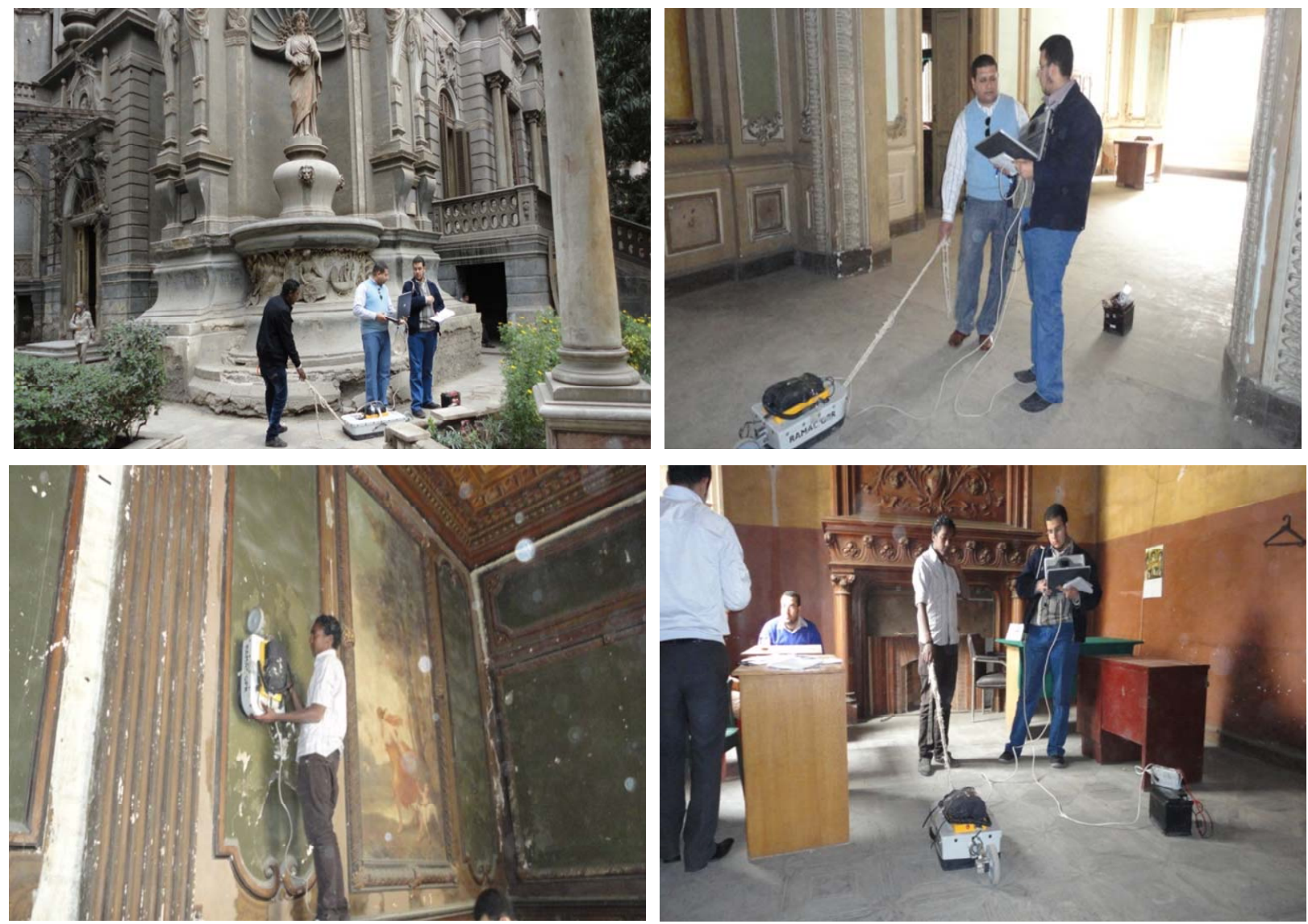

Figure 2. Investigations of the Sakakini palace in Cairo.
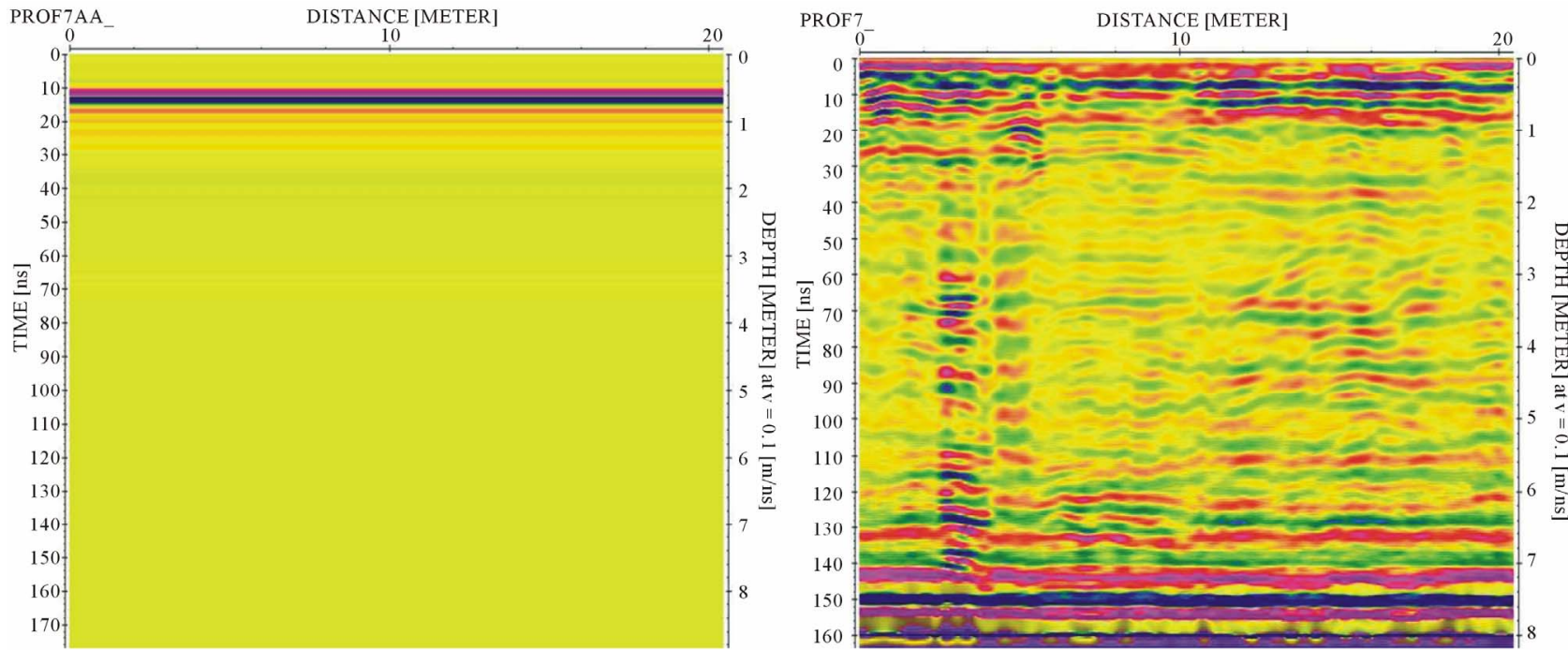

Figure 3. Effect of the processing sequence on the raw GPR data.

Interpretation of survey data must be a cooperative process involving both archaeological geophysicists and archaeologists that are familiar with the specific cultural context of the site being studied. An understanding of the geological context of the survey area is also very important.

\section{Results of GPR at the Basement of the Palace}

About 50 GPR profiles conducted at the basement floor. The target of these profiles is to depict the subsurface condition concerning the layering, disturbed and col- 
lapsed soils and foundation locations. Figures 4-7 show the radar profile No. 7, 8, 22 and 29 and shows a disturbed soil and the expected locations of the foundation at depth of about 3 meters.

The soil succession shows a fill below the floor extended to about 5 to 7 meters. The fill consists of silt, clays and some stone fragment in some localities. The silt become more clayey below 4 meter depth as indicated from the amplitude of radar reflection. Shallow water table might be due to the seepage or the past history of the lagoon area at the site of the Palace. The locations of the interpreted profiles in the basement floor are shown in (Figure 8).

\section{Results of GPR at Palace Surrounding}

About 30 GPR profiles conducted at the area surrounding the palace. The target of these profiles is to depict the subsurface condition concerning the layering, water table, disturbed and collapsed soils and foundation locations.

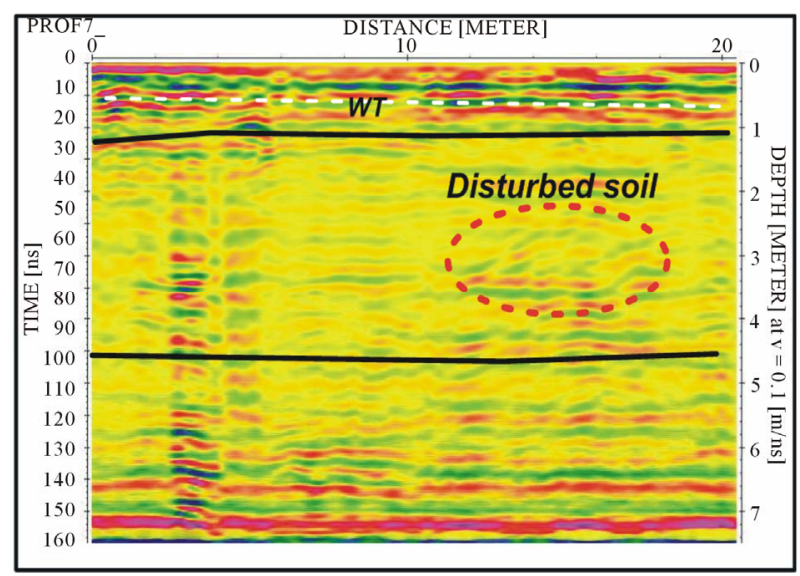

Figure 4. Interpreted GPR profile No. 7 at the basement area showing the disturbed soil at depth about $3 \mathbf{m}$.

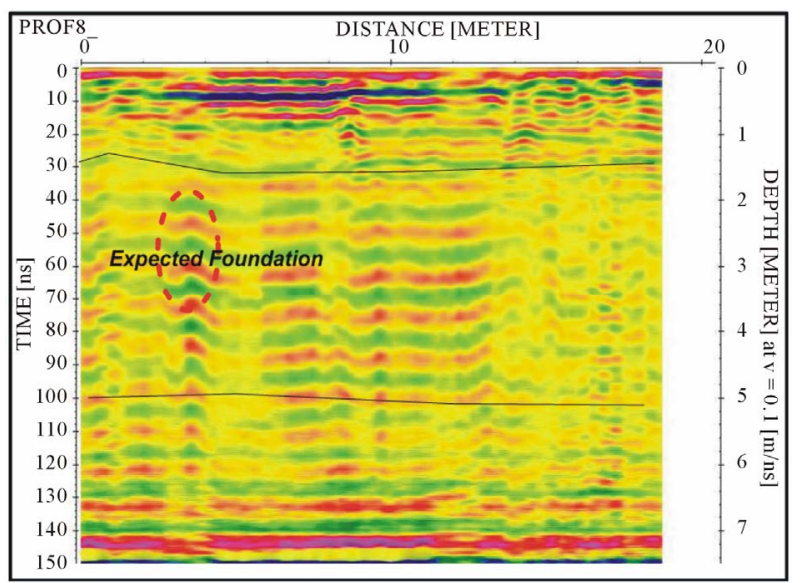

Figure 5. Interpreted GPR profile No. 8 at the basement area showing the expected foundation at depth about 2.5 to $3 \mathbf{m}$.

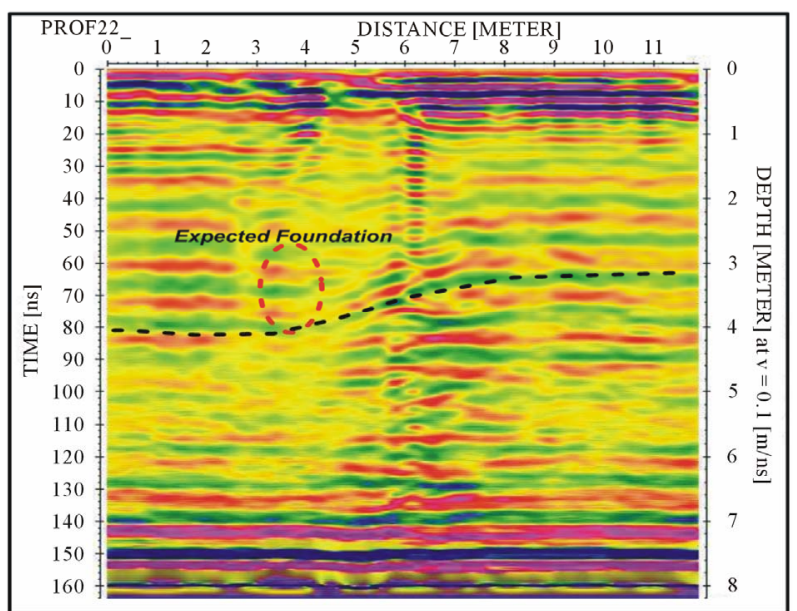

Figure 6. Interpreted GPR profile No. 22 at the basement area showing the expected disturbed soil due to foundation at about $3 \mathrm{~m}$ depth.

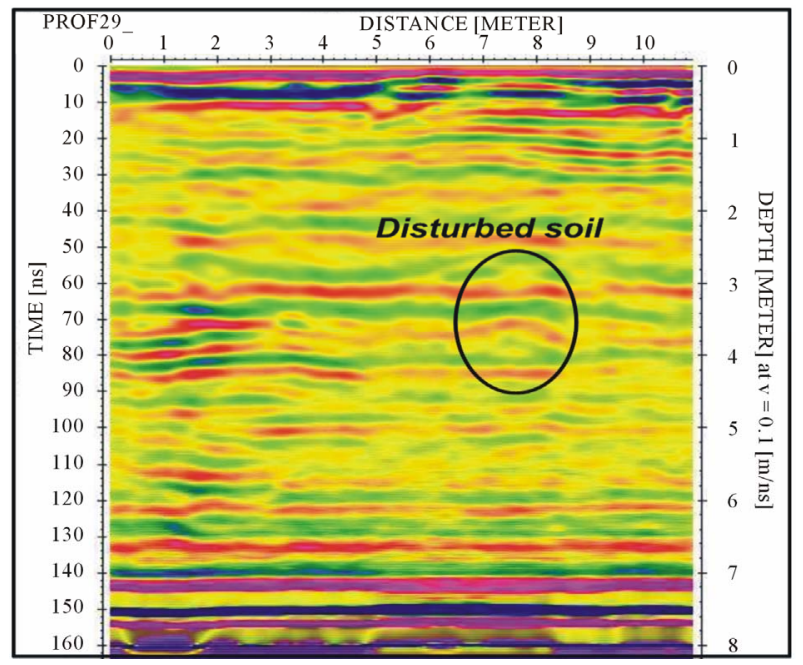

Figure 7. Interpreted GPR profile No. 29 at the basement area showing the expected foundation at depth about $\mathbf{3}$.

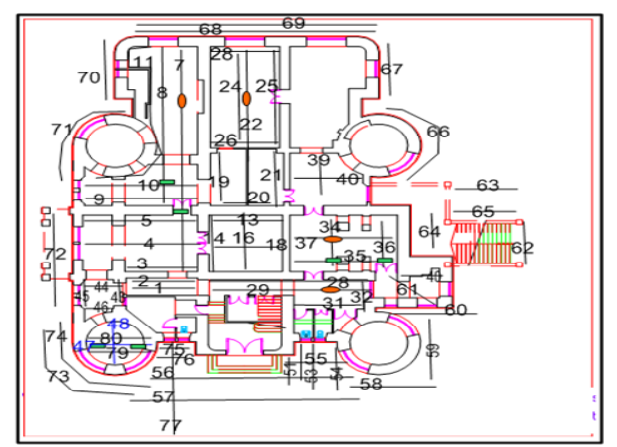

Figure 8. Interpreted locations of interrupted soils (red circles) and expected foundation (green box) in basement area.

(Figures 9-14), show the radar profiles No. 56, 57, 61, $66,71,74$, and 75 and shows a disturbed soil and the 


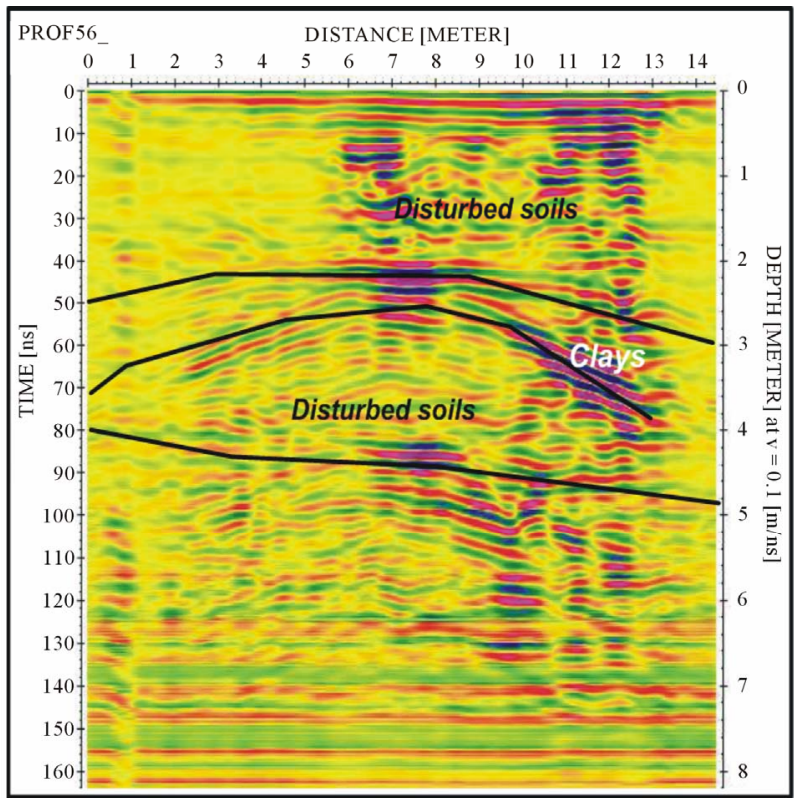

Figure 9. Interpreted GPR profile No. 56 at the basement area showing the expected disturbed soils near the tower.

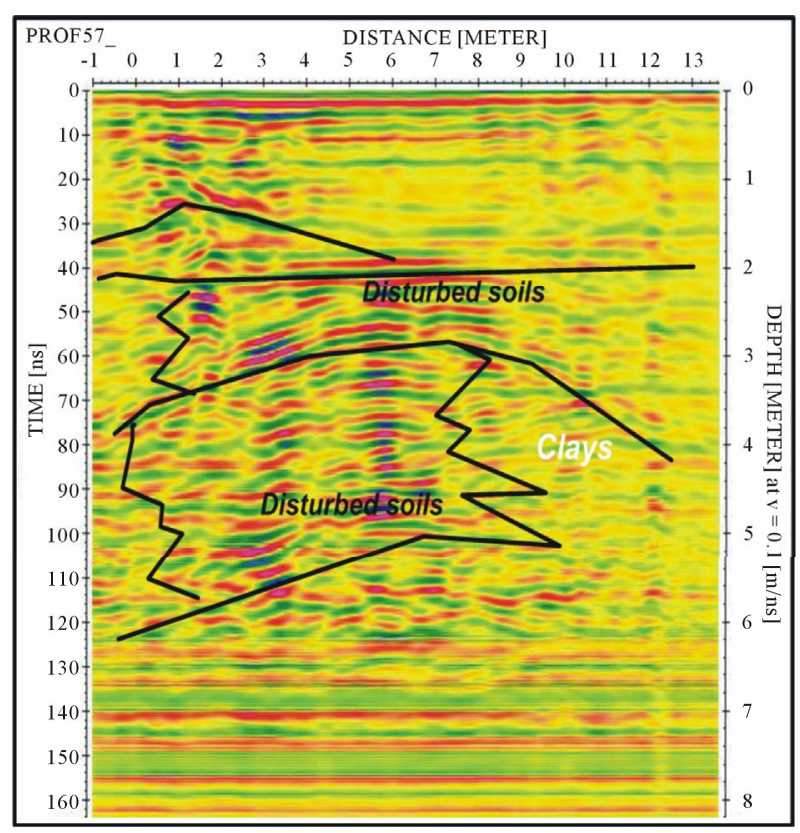

Figure 10. Interpreted GPR profile No. 57 at the basement area showing the expected disturbed soils near the tower.

expected locations of the foundation at depth of about 3 meters.

The soil succession shows a fill below the ground surface extended to about 7 meters. The fill consists of silt, clays and some stone fragment in some localities. The silt become more clayey below 4 meter depth as indicated from the amplitude of radar reflection. Shallow water table might be due to the seepage or the past history of the lagoon area at the site of the Palace. Some

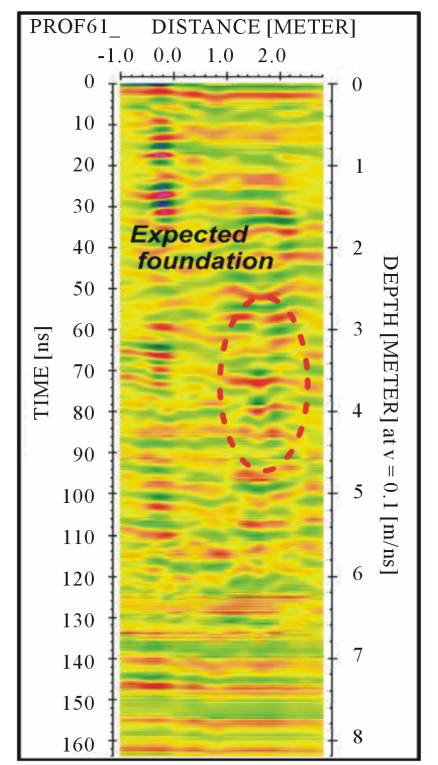

Figure 11. Interpreted GPR profile No. 61 at the surrounding area showing the expected foundations.

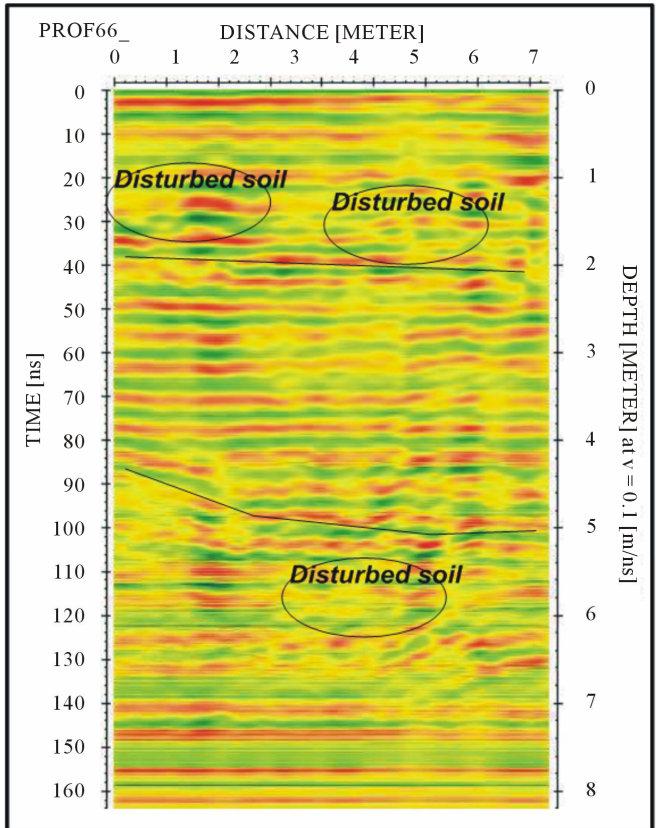

Figure 12. Interpreted GPR profile No. 66 at the surrounding area showing the expected disturbed soils near the tower.

profiles show up-arching of the soil layers and lateral inhomogenety of the soil composition. The locations of the interpreted profiles in the basement floor are shown in (Figure 15).

\section{Results of GPR Surveys at Palace Walls and First Floor}

About 20 GPR profiles conducted at some walls at the 


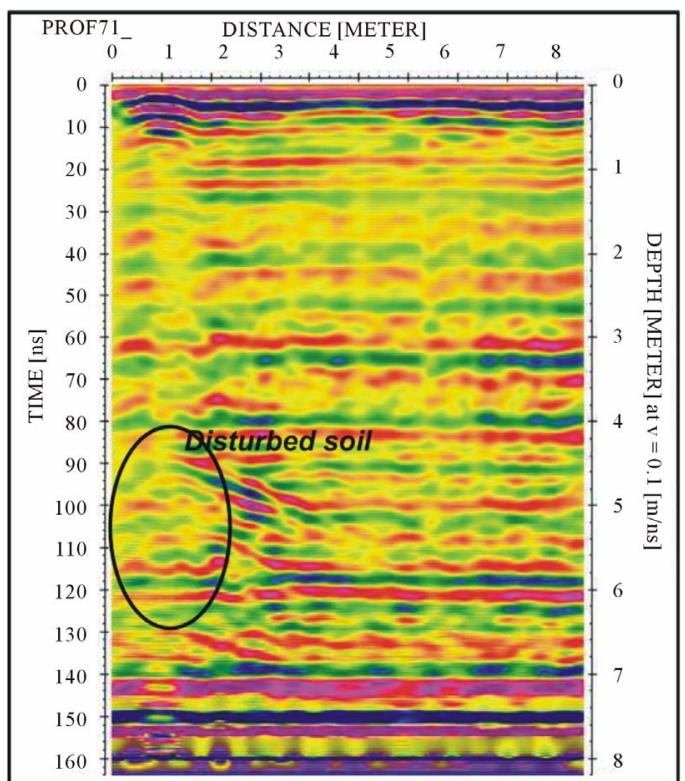

Figure 13. Interpreted GPR profile No. 71 at the surrounding area showing the expected disturbed soils near the tower.

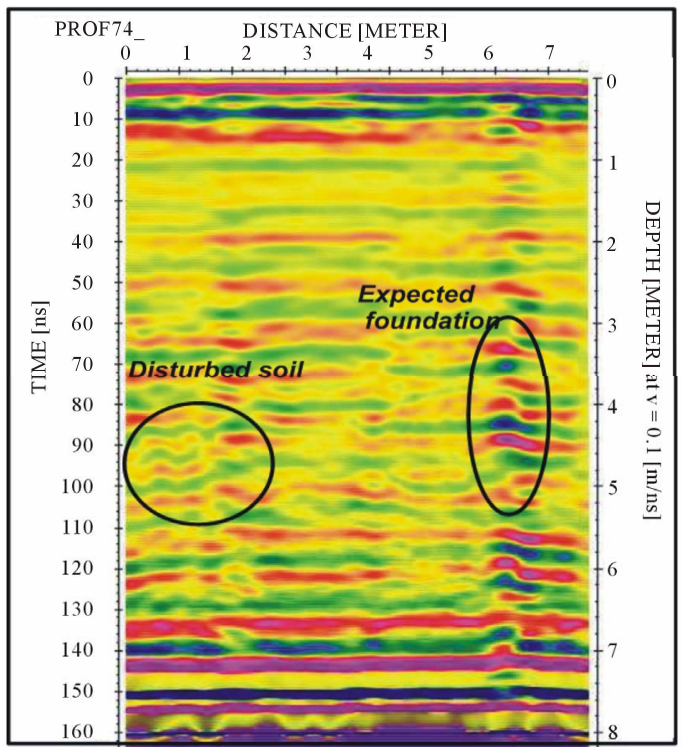

Figure 14. Interpreted GPR profile No. 74 at the surrounding area showing the expected disturbed soils near the tower.

palace. The target of these profiles is to depict the subsurface condition concerning the layering, water table, disturbed and collapsed soils and foundation locations. (Figures 16-20), show the radar profiles No. 81, 85, 88, 89, and 99. These profiles indicates the fractures and cracks both in walls and concrete slabs as well as the variation of the ground of the first floor.

\section{Conclusions}

The analysis of the geophysical dataset obtained at the

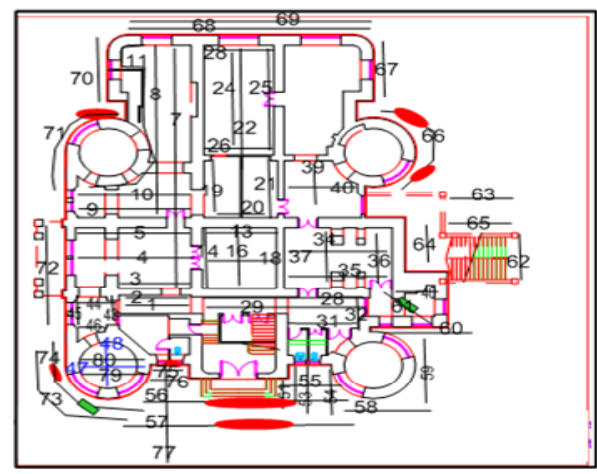

Figure 15. Interpreted locations of interrupted soils (red circles) and expected foundation (green box), surrounding area.

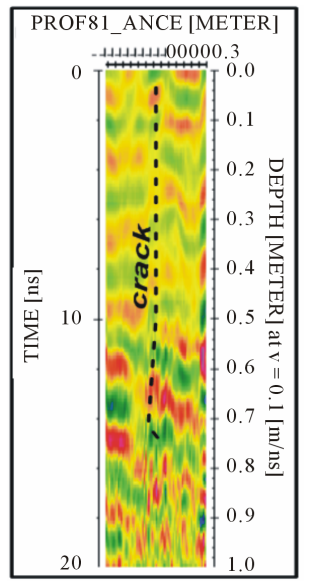

Figure 16. Interpreted GPR profile No. 81 at the outside wall near elevator door.

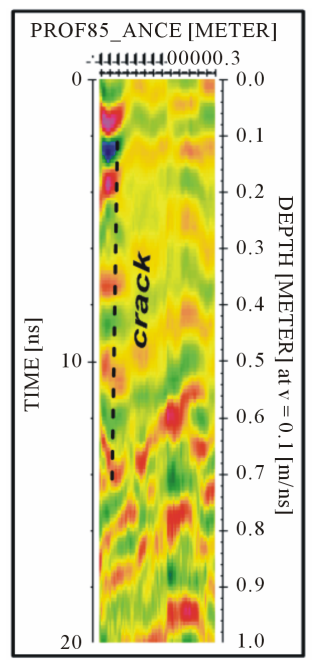

Figure 17. Interpreted GPR profile No. 85 at the outside tower right side of elevator door.

palace of Habib Sakakini site gives evidences of the excellent performance of integrated geophysical and geotechnical techniques for the identification and mapping 


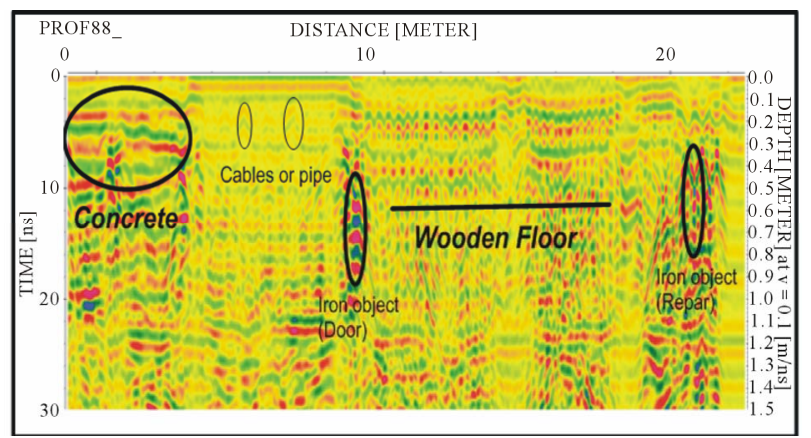

Figure 18. Interpreted GPR profile No. 88 at the ground of the first floor.

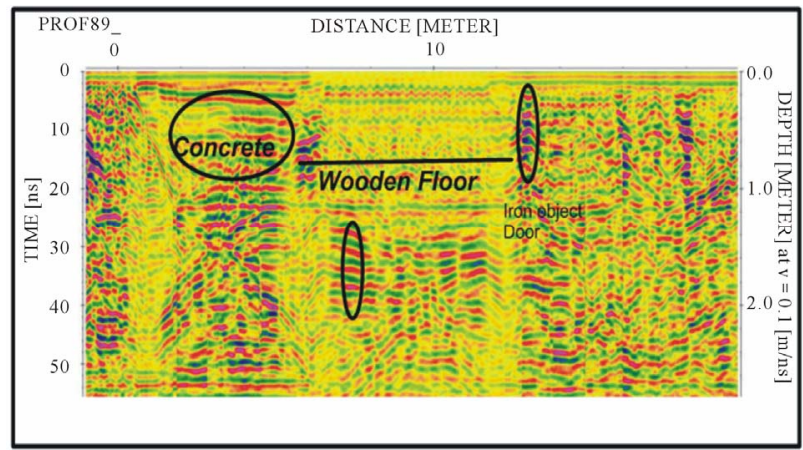

Figure 19. Interpreted GPR profile No. 89 at the ground of the first floor.

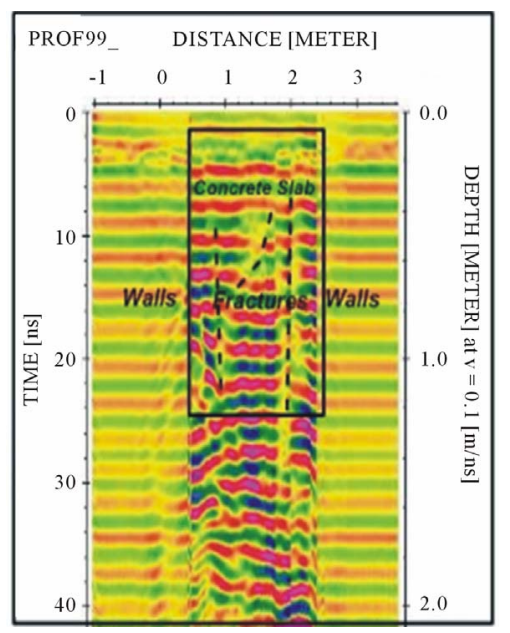

Figure 20. Interpreted GPR profile No. 99 at the slab with fractures at Basement floor.

of low-contrast buried cultural heritage and underground, soil-foundation conditions and interactions, GWT and structural conditions and state of deterioration and cracking. As well as the floors and ceilings condition and structural mapping. MF GPR provide an effective solutions to detect targets, which exhibit small differences in physical properties with references to the surrounding sediments due to their high sensitivity and resolution. GPR vertical and lateral resolution is decimetric and adequate for the objective of the survey. Depth penetration is in the range between 150 and $500 \mathrm{~cm}$, on the average adequate for the objective of survey. The variations depend on local soil conditions and particularly on lenses of inorganic and organic materials, which are imaged by GPR. Depth calibration was performed by means of MF GPR data analysis and validation at the soil face exposed by the geotechnical surveys. GPR is a adequate to image the shallow 3 to 5 meters. Information concerning deeper targets can be extrapolated from the detailed reconstruction of the deformation of the shallow layers. The profiles obtained by means of MF methods actually exhibit extended depth range due to substantial signal-to-noise ratio enhancement in the deepest part of the record.

Several targets of potential engineering interest were identified from the integrated GPR and geotechnical survey data and interpretations.

- The soil column of the site comprises heterogeneous materials as a fill for about 5 to 7 meters.

- The shallow ground water table, about 0.5 - 1 meter, has a serious influence on the rigidity of the soils and imposes humidity to the foundations and walls of the Palace.

- The composition of silt and clay soil for great depth might affect the stability of the site.

- Many fractures detected in walls and concrete slabs that extended to about $60 \mathrm{~cm}$ in the walls and slabs.

- The foundation type is shallow strip and spread stone foundations at depth of 2.5 to 3 meters on concrete raft or mat.

- The wall masonry construction system is multiple leaf masonry walls or rubble infill walls.

- The added court in the eastern side is concrete structure.

\section{Acknowledgements}

The present paper is part of project entitled "risk assessment and seismic response analysis of architectural heritage in Egypt” funded by Cairo University and under the management of Dr Sayed Hemeda, the author and main investigator of the project. I gratefully acknowledge the support and assistance of professor Sharaf el-din Mahmoud, the chairman of geophysics Department, Cairo University for providing the open source interpretation software used for 3-D data analysis and his great efforts and help through the investigation.

\section{REFERENCES}

[1] H. Becker and J. W. E. Fassbinder "Magnetic Prospecting in Archaeological Sites, Monuments and Sites, VI,” ICOMOS, Paris, 2001.

[2] A. T. Batayneh, A. A. Abueladas and K. A. Moumani, "Use of Ground Penetrating Radar for Assessment of Po- 
tential Sinkhole Conditions: An Example from Ghor al Haditha Area, Jordan,” Environmental Geology, Vol. 41, No. 8, 2002, pp. 977-983.

[3] L. B. Conyers and D. Goodman, "Ground Penetrating Radar: An Introduction for Archaeologists,” Altamira Press, Walnut Creek, 1997.

[4] A. P. Annan and S. W. Cosway, "Ground Penetrating Radar Survey Design," The Annual Meeting of SAGEEP, Chicago, 26-29 April 1992.

[5] B. W. Bevan, “The Search for Graves,” Geophysics, Vol. 56, No. 9, 1991, pp. 1310-1319.

[6] J. Mellet, "Location of Human Remains with Ground Penetrating Radar,” Special Paper No. 16, Geological Survey of Finland, Espoo, 1992.

[7] E. Forte and M. Pipan, "Integrated Seismic Tomography and ground Penetrating Radar (GPR) for the High Resolution Study of Burial Mounds (Tumil)," Journal of Archaeological Science, Vol. 35, No. 9, 2008, pp. 2614-2623. doi:10.1016/j.jas.2008.04.024

[8] C. J. Vaughan, "Ground Penetrating Radar Surveys Used in archaeological Investigations," Geophysics, Vol. 51, No. 3, 1986, pp. 595-604.
[9] D. Goodman, Y. Nishaimura and J. D. Rogers, "GPR Time-Slices in Archaeological Prospection,” Archaeological Prospection, Vol. 2, No. 2, 1992, pp. 85-89.

[10] M. Pipan, I. Finetti, A. Del Ben and F. Ferigo, "Recent Advances in the Multifold and 3D GPR Exploration of the Aquileia Archaeological Park (Italy)," The 58th Conference of European Association of Geoscientists \& Engineers, Paper M020, Amsterdam, 1996.

[11] M. Pipan, I. Finetti and F. Ferigo, "Multi-Fold GPR Techniques with Applications to High-Resolution Studies: Two Case Histories," European Journal of Environmental and Engineering Geophysics, Vol. 1, No. 1, 1996, pp. 83-103.

[12] E. Forte, M. Pipan and A. Del Ben, "Integrated Geophysical Survey of the Medinet Madi Archaeological Site (Fayoum, Egypt)," Proceeding of the 4th International Congress on "Science and Technology for the Safeguard of Culture Heritage in the Mediterranean Basin”, Cairo, 6-8 December 2009, pp. 214-218.

[13] A. P. Annan, "Practical Processing of GPR Data," Sensors and Software, Ontario, 1999.

[14] K. J. Sandmeier, "ReflexW,” Version 4.0, Sandmeier Scientific Software, Karlsruhe, 2006. 